Sharif University of Technology
Scientia Iranica
SCIENTIA

\title{
Effects of shock wave/boundary-layer interaction on performance and stability of a mixed-compression inlet
}

\author{
M.R. Soltani ${ }^{\mathrm{a}, *}$, A. Daliri ${ }^{\mathrm{a}}$ and J. Sepahi Younsi ${ }^{\mathrm{b}}$ \\ a. Department of Aerospace Engineering, Sharif University of Technology, Tehran, P.O. Box 1458889694, Iran. \\ b. Faculty of Mechanical \& Aerospace Engineering, Ferdowsi University of Mashhad, Mashhad, Iran. \\ Received 24 January 2015; received in revised form 8 May 2015; accepted 26 October 2015
}

\author{
KEYWORDS \\ Supersonic inlet; \\ Shock \\ wave/Boundary-Layer \\ Interaction (SBLI); \\ Pseudo-shock; \\ Shock train; \\ Buzz.
}

\begin{abstract}
Experiments were conducted to study various kinds of Shock wave/Boundary Layer Interaction (SBLI) in an axisymmetric mixed-compression inlet. Experimental findings were compared and verified by numerical solutions where possible. Different types of SBLI relevant to the mixed-compression inlets are classified. Interactions of normal shock wave/boundary-layer at subcritical condition and in buzz condition are investigated using Schlieren and shadowgraph flow visualization as well as unsteady pressure recordings. The data is compared with the CFD predictions. Interactions of cowl lip reflected oblique shock and the terminal normal shock with the spike boundary-layer at both critical and supercritical operations, which lead to pseudo-shock phenomena, are also studied. Experimental pressure recordings are used for validation and discussion. For near-critical throttling values, interaction of internal compression oblique shocks with boundary-layer and pseudo-shock phenomenon is dominant. Formation of lambda shock due to interaction of separated boundary-layer with normal shock wave is investigated. Each type of flow interaction phenomena has different effects on the stability and performance of the inlet. Interaction of terminal normal shock with internal duct boundary-layer causes pseudoshock phenomenon that increases flow distortion and reduces total pressure recovery. Interaction of normal shock with cone boundary-layer causes buzz instability and degrades inlet performance.
\end{abstract}

(C) 2016 Sharif University of Technology. All rights reserved.

\section{Introduction}

For supersonic vehicles, the inlet must decelerate the incoming flow to subsonic speeds before delivering it to the combustion chamber. The compression could be done by a normal shock or by a series of oblique shocks. The latter case produces lower entropy changes and is frequently used. Depending on whether the oblique shock waves are generated inside or outside the inlet duct, such designs are referred to as internal and external compression inlets, respectively. Both external and internal types of compression are also used

\footnotetext{
*. Corresponding author.

E-mail address: msoltani@sharif.edu (M.R. Soltani)
}

in appropriate degrees to reduce their deficiencies in another design known as mixed compression. In all cases, interaction of shock wave with inlet surface boundarylayer is inevitable. Oblique shock waves interact with boundary-layer and cause adverse pressure gradient that makes the boundary-layer ready for separation. A final near-normal shock wave (or an oblique shock wave of strong-shock solution) turns the flow from supersonic to subsonic through a strong transonic SBLI and will usually separate the flow. Flow separation greatly impacts the inlet performance by means of reducing the total pressure recovery and by adding flow distortion. As the flow is typically separated at the entrance of subsonic diffuser, it becomes nonuniform at the engine face [1]. This flow distortion 
disturbs inlet performance and harms the engine. The terminal normal shock can be expelled out of the inlet duct by reducing the inlet mass flow ratio (at subcritical operating condition); however, the progress will result in the shock interaction with the boundarylayer of the external compression surface that will lead to sudden high-amplitude flow oscillations called buzz. In addition to degrading the inlet performance, buzz can lead to trust loss, engine surge, or even aircraft structural damages [2].

Numerous studies have been carried out on supersonic inlet performance and stability since 1940 . Much information could be found in the literature on different aspects of various types of supersonic inlet models [3-14]. Performance characteristics of inlet in cases of extensive maneuvering range [3,4], unstart [5], buzz phenomenon, and application of boundary-layer bleed [6-14] have recently been investigated. In addition to these experiments, many computational studies have been accomplished on the supersonic inlet flow and buzz phenomenon as well as flow control methods [15-19].

Shock wave boundary-layer interaction as a fundamental gas dynamics phenomenon can be found in many practical aerospace related problems ranging from transonic aircraft wings to hypersonic vehicles and engines. A comprehensive survey of the interaction of shock wave and turbulent boundary layer was published by Viswanath in 1988 [20]. In his paper, an overview of some developments in understanding, prediction, and control of two-dimensional SBLI at high speeds was presented. A general review of different aspects of SBLI along the wall surface of internal compressible flows was presented by Matsuo et al. [21]. In this study, the fundamental feature of shock train and pseudo-shock, several prediction methods, and control methods of pseudo-shock were described. Also, understandings related to self-excited oscillations of pseudo-shock were reviewed. A summary of the Computational Fluid Dynamics (CFD) simulations of SBLI was also presented in a review paper and methods of DNS, LES, and RANS were compared with experiments and the capabilities and limitations of each turbulent model were described [22]. Also, application of LES method for simulation of shock wave interactions in nozzles was performed [23,24]. Recently, comprehensive study on the advances of SBLI with emphasis on flow unsteadiness, heat transfer prediction, multi-shock boundary-layer interaction, and flow control techniques has been performed [25]. Other recent studies have been carried out on unsteadiness of SBLI $[26,27]$ and control methods such as bleed, vortex generator, and micro actuators [28-30]. Also, some recent experiments and theoretical efforts have been made to investigate the effect of flow control methods such as bleed on SBLI for supersonic and hypersonic inlets [31-35]. The main idea of these notable papers is to discuss the most general aspects of shock wave boundary-layer interactions relevant to supersonic air inlets.

Studying the physics of complicated flow corresponding to SBLI in order to employ the results for designing inlets and related flow control devices is useful. Although several studies have been performed on SBLI and its control means, some of the complicated aspects of SBLI are not discovered yet and it is needed to perform more experiments and simulations to perceive the physics of flow interactions. On the other hand, generalization and application of the fundamental SBLI flows for practical flow devices such as inlets are very useful and could hardly be founded in the literature. Along with classification of SBLI flows relevant to mixed-compression inlets, this paper aims to present a novel analysis to relate SBLI phenomena to inlet performance and stability through evaluation of experimental and numerical evidences.

\section{Experimental setup}

\subsection{Wind tunnel}

The experiments are conducted in a continuous suction-type wind tunnel with a rectangular test section of $60 \times 60 \mathrm{~cm}^{2}$. The tunnel is equipped with a flexible nozzle that can be adjusted during the tests. The turbulence intensity of the flow in the test section ranges from $0.4 \%$ to $1.4 \%$, depending on the free-stream Mach number [12]. There exist porous bleed holes on the upper and lower walls of the test section which can stabilize and control wind tunnel shock and other reflected waves. Side wall windows of the test section have been made up of optical glasses which allow the flow and shock pattern observation by means of Schlieren and shadowgraph flow visualization systems. The tunnel is indraft; thus, total pressure and total temperature in the test section are constant and atmospheric.

\subsection{Model}

Figure 1(a) shows the inlet model used in the experiments. It is an axisymmetric mixed-compression

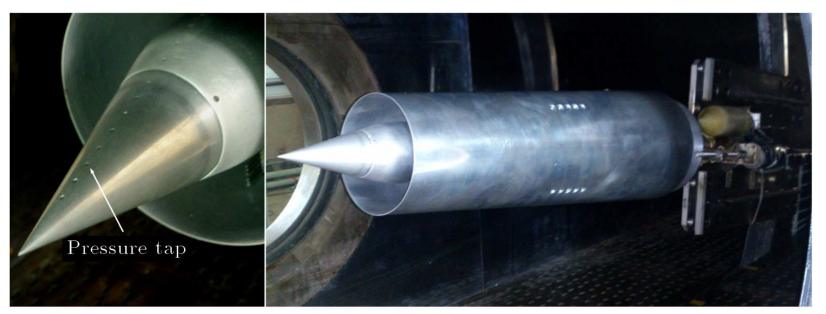

(a)

(b)

Figure 1. (a) Inlet model in wind tunnel. (b) Tip cone and pressure taps. 


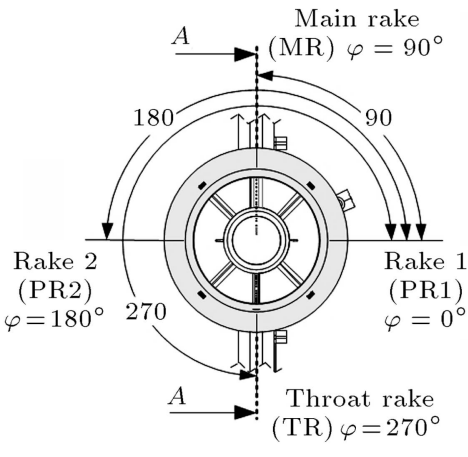

(a)

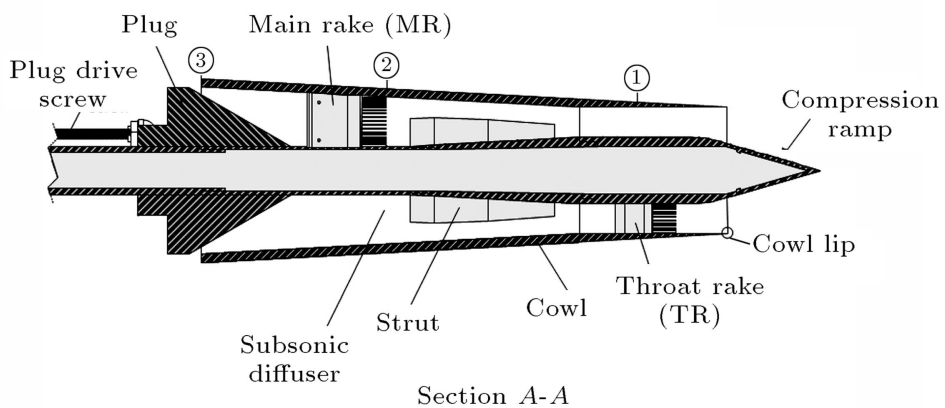

(b)

Figure 2. Schematic of the intake model and its instruments.

Table 1. Measurement inaccuracies (\%).

\begin{tabular}{cccccc}
\hline $\boldsymbol{\Delta} \mathrm{M}_{\infty} / \mathrm{M}_{\infty}$ & $\boldsymbol{\Delta} \boldsymbol{P}_{\mathbf{0} \infty} / \boldsymbol{P}_{\infty}$ & $\boldsymbol{\Delta} \operatorname{Re} / \operatorname{Re}$ & $\boldsymbol{\Delta} \boldsymbol{P} / \boldsymbol{P}$ & $\boldsymbol{\Delta} \varepsilon / \varepsilon$ & $\boldsymbol{\Delta} \boldsymbol{\eta} / \boldsymbol{\eta}$ \\
\hline 1.458 & 0.012 & 1.982 & $0.929,1.421$ & 2.418 & 1.421 \\
\hline
\end{tabular}

inlet with design Mach number of 2.0 and $L / d=3.4$. Bleed could be added or removed by replacing tip cones (Figure 1(b)). However, in this study, no bleed is applied. A conical plug is located at the end of the model to change the exit area of the inlet during the tests. The plug translates along the intake centerbody via a DC motor and a ball screw (Figure 2(b)). The inlet mass flow rate and back pressure ratio are controlled through changing the exit area. Note that the back pressure determines the normal shock position and consequently design and off-design conditions of the intake can be obtained.

\subsection{Pressure transducers and test procedure}

Sixty two sensitive pressure transducers have been used to measure static and total pressures on the model and wind tunnel walls. Several pressure taps have been located on different positions of the spike surface to measure the static pressure distribution. Two multi-probe rakes, TR (Throat Rake) and MR (Main Rake) as shown schematically in Figure 2, have been located at the throat $(x / d=0.8$ and $\phi=$ $\left.270^{\circ}\right)$ and at the exit $\left(x / d=2.4\right.$ and $\left.\phi=90^{\circ}\right)$ sections of the intake with blockage ratios of $2 \%$ and $4.3 \%$, respectively. Rake TR has 12 probes and is used to measure the boundary-layer profile and flow behavior at the throat section. Rake MR has 17 probes and is used to measure the boundary-layer profile, inlet total pressure recovery, mass flow rate, and flow distortion at the exit face of the model. Two single-probe rakes (PR1 and PR2) are also located at $x / d=1.4-\phi=0^{\circ}$ and at $x / d=1.8-\phi=$ $180^{\circ}$, respectively, for total pressure losses measurement. All sensors have accuracy of $\pm 0.1 \%$ of full scale and a corresponding natural frequency response of $150 \mathrm{KHz}$. An accurate industrial data acquisition board is used. The frequency of measurement is $2800 \mathrm{~Hz}$.

The intake has been tested at a free-stream Mach number of 2.0 without implementation of the bleed. All tests were conducted at zero-degree angle of attack. At the beginning of each test, the plug was in the rear position (fully open exit). Then, the plug was translated forward to reduce the exit area. For every free-stream Mach number, a similar set of eight exit areas was adjusted during the tests and pressures at sixty two points were obtained.

Measurement inaccuracies of some important values are listed in Table 1 . Two values have been reported for static pressure since two different types of pressure transducers have been used in this work.

\subsection{Flow visualization setup}

In this experiment, both Schlieren and shadowgraph optical techniques were used to visualize shock pattern and flow structure on the external region of the inlet. Mirrors and light source were arranged in a Z-type configuration [36] and an accurate table with two degrees of freedom was used to locate the knife-edge (razor blade in this case) at the focal of the receiving part. A CCD camera with a recording speed of 1000 frames per second (fps) was also used for taking the pictures.

\section{Numerical method}

A Computational Fluid Dynamics (CFD) solver developed by authors was used to simulate the flow inside the inlet at its design free-stream Mach number, $M_{\infty}=2.0$, in all operating conditions. In this code, ReynoldsAveraged Navier-Stocks (RANS) equations are solved numerically. Neglecting body forces, these equations, 
in two-dimensional conservative forms, are:

$$
\frac{\partial Q}{\partial t}+\frac{\partial T}{\partial x}+\frac{\partial U}{\partial y}+\alpha V=\frac{\partial T_{v}}{\partial x}+\frac{\partial U_{v}}{\partial y}+\alpha V_{v}+S
$$$$
Q=\left[\begin{array}{c}
\rho \\
\rho u \\
\rho \nu \\
\rho E \\
\rho k \\
\rho \omega
\end{array}\right], \quad T=\left[\begin{array}{c}
\rho u \\
P+\rho u^{2} \\
\rho u \nu \\
\rho u H \\
\rho u k \\
\rho u \omega
\end{array}\right]
$$$$
U=\left[\begin{array}{c}
\rho v \\
\rho u v \\
P+\rho \nu^{2} \\
\rho v H \\
\rho v k \\
\rho v \omega
\end{array}\right], \quad V=\frac{1}{y}\left[\begin{array}{c}
\rho v \\
\rho u v \\
\rho \nu^{2} \\
\rho v H \\
\rho v k \\
\rho v \omega
\end{array}\right],
$$$$
T_{v}=\left[\begin{array}{c}
0 \\
\tau_{x x} \\
\tau_{x y} \\
u \tau_{x x}+v \tau_{x y}-q_{x} \\
\left(\mu_{L}+\sigma_{k} \mu_{t}\right) \frac{\partial k}{\partial x} \\
\left(\mu_{L}+\sigma_{\omega} \mu_{t}\right) \frac{\partial \omega}{\partial x}
\end{array}\right]
$$$$
T_{v}=\left[\begin{array}{c}
0 \\
\tau_{x x} \\
\tau_{x y} \\
u \tau_{x y}+v \tau_{y y}-q_{y} \\
\left(\mu_{L}+\sigma_{k} \mu_{t}\right) \frac{\partial k}{\partial x} \\
\left(\mu_{L}+\sigma_{\omega} \mu_{t}\right) \frac{\partial \omega}{\partial x}
\end{array}\right]
$$

$$
T_{v}=\left[\begin{array}{c}
0 \\
\tau_{x r} \\
\tau_{r r}-\tau_{\theta \theta} \\
u \tau_{x y}+v \tau_{y y}-q_{y} \\
\left(\mu_{L}+\sigma_{k} \mu_{t}\right) \frac{\partial k}{\partial x} \\
\left(\mu_{L}+\sigma_{\omega} \mu_{t}\right) \frac{\partial \omega}{\partial x}
\end{array}\right]
$$

where $Q$ denotes the conservative variable vector, $T$ and $U$ are inviscid flux vectors, $T_{v}$ and $U_{v}$ are the viscous flux vectors in each spatial direction, $V$ and $V_{v}$ are the source terms associated with the asymmetry, and $S$ is the source term due to the turbulence model equations.

For a 2D axisymmetric problem, let $\alpha=1$. Using explicit finite volume discretization, Eq. (1) becomes:

$$
\begin{aligned}
Q_{i, j}^{n+1}= & Q_{i, j}^{n}-\frac{\Delta t_{i, j}}{A_{i, j}}\left[\sum_{k=1}^{4}\left(F_{c}\right)_{k} \Delta s_{k}\right]-\alpha \Delta t_{i, j} V_{i, j} \\
& +\frac{\Delta t_{i, j}}{A_{i, j}}\left[\sum_{k=1}^{4}\left(F_{v}\right)_{k} \Delta s_{k}\right]+\alpha \Delta t_{i, j} V_{v i, j},
\end{aligned}
$$

where:

$$
\begin{aligned}
& F_{c}=\left[\begin{array}{c}
\rho q \\
\rho u q+n_{x} P \\
\rho v q+n_{y} P \\
\rho u H
\end{array}\right], \quad F_{v}=\left[\begin{array}{c}
0 \\
n_{x} \tau_{x x}+n_{y} \tau_{x y} \\
n_{x} \tau_{x y}+n_{y} \tau_{y y} \\
n_{x} \Theta_{x}+n_{y} \Theta_{y}
\end{array}\right] \\
& \Theta_{x}=u \tau_{x x}+v \tau_{x y}+k \frac{\partial T}{\partial x} \\
& \Theta_{x}=u \tau_{x y}+v \tau_{y y}+k \frac{\partial T}{\partial y}
\end{aligned}
$$

In the above equations, $H$ is the total enthalpy, $A$ is the area of the cell, $\Delta s$ is the length of the cell face, and $q$ is the velocity component normal to the cell face. The convective fluxes are computed by the second-order accurate Roe scheme. Also, a finite volume method, consistent with the overall discretization method, is used to calculate viscous fluxes. Further descriptions of the governing equations and the numerical considerations could be found in $[37,38]$.

Viscosity coefficient is calculated using the linear interpolation of laminar and turbulent viscosity coefficients. Laminar viscosity coefficient is molecular viscosity and it has been calculated using Sutherland relation. The turbulent viscosity coefficient, however, has been calculated by the Menter's two-equation eddy viscosity $k-\omega$ SST turbulence model [38]. The eddy viscosity of SST model is defined by [39]:

$$
v_{T}=\left(a_{1} k\right) / \max \left(a_{1} \omega ; \Omega F_{2}\right) .
$$

With $a_{1}$ being a constant, $\Omega$ equals the term $\partial u / \partial y$ and $F_{2}$ is a function that is one for boundary-layer flows and zero for free shear layers [39].

The grid used for the simulations is shown in Figure 3. This grid was generated by an elliptic grid generator and could be refined or stretched in all or part of the physical domain. This structured grid is refined at the cowl lip and at the internal walls in order to capture physics of the boundary layer flow.

An intensive grid resolution study was performed to ensure the independence of the numerical solution from the grid size. Finally, a fine grid with about 90000

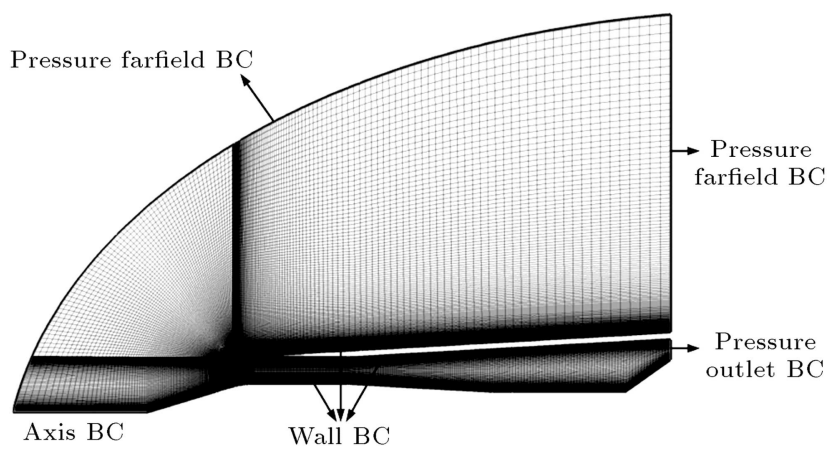

Figure 3. Elliptic grid around the inlet of study and boundary conditions. 


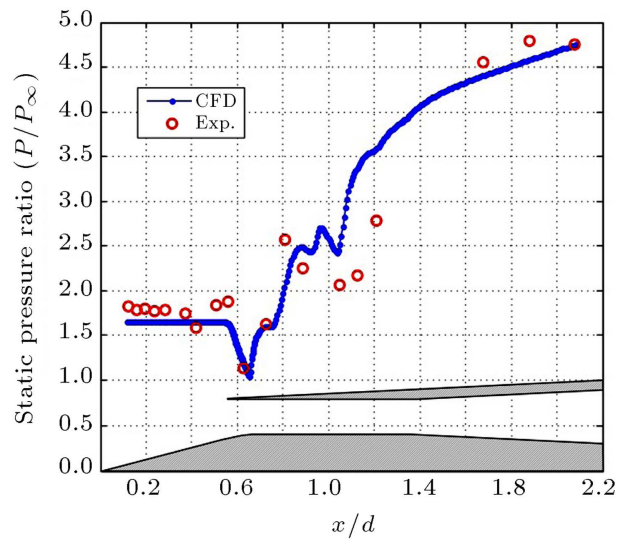

(a)

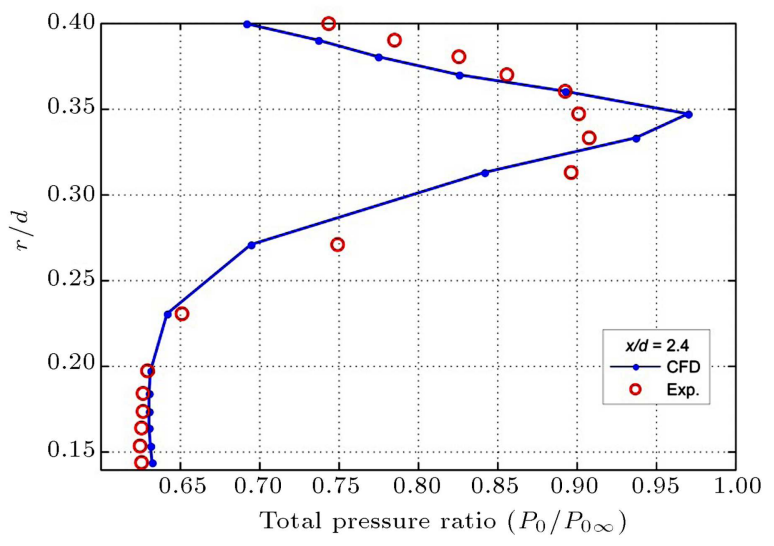

(b)

Figure 4. Comparison of the present numerical prediction with experimental pressure recordings, $M_{\infty}=2.0, \alpha=0$, and $\mathrm{EBR}=60 \%$ : (a) Static pressure ratio distribution on the spike; and (b) radial distribution for total pressure ratio at $x / d=2.4$.

cells was chosen as the most optimum one. Further, near the wall, turbulent flow behavior is considered and the first node (or cell centroid) is chosen at $y^{+}$equal to unity.

Figure 4(a) compares the numerical and experimental static pressure distributions on the spike for Exit Blockage Ratio (EBR) of $60 \%$ and at a free-stream Mach number of 2.0 at zero-degree angle of attack. It could be seen that relatively good agreement between numerical prediction and experimental data exists. The error seen in the region of throat may be due to the effect of rake TR and struts blockage that have not been taken into account in the numerical prediction. However, maximum error in this region does not exceed $25 \%$. Total pressure ratio at the subsonic diffuser is measured by the rake $\mathrm{MR}$ at $x / d=2.4$ and is compared with the numerical prediction in Figure 4(b). Again, good agreement between the experimental and numerical data is achieved. The profile of Figure 4(b) further ensures that the extent of flow separation has been simulated with acceptable accuracy.

Figure 5 compares the axial density gradient contour together with shadowgraph shock structure visualization for a free-stream Mach number of 2.0 at zero-degree angle of attack for a supercritical condition to be defined in the next section. It can be seen that the location and inclination of shock waves have been simulated precisely. It also verifies good performance of the numerical solver.

\section{Inlet operating regimes}

This section will remind different operating regimes of mixed-compression inlet under study and the corresponding shock patterns. Characteristic curve of an inlet, as shown in Figure 6, demonstrates variation of total pressure recovery of inlet versus inlet mass flow ratio. Total pressure recovery of inlet, $\eta$, is defined as

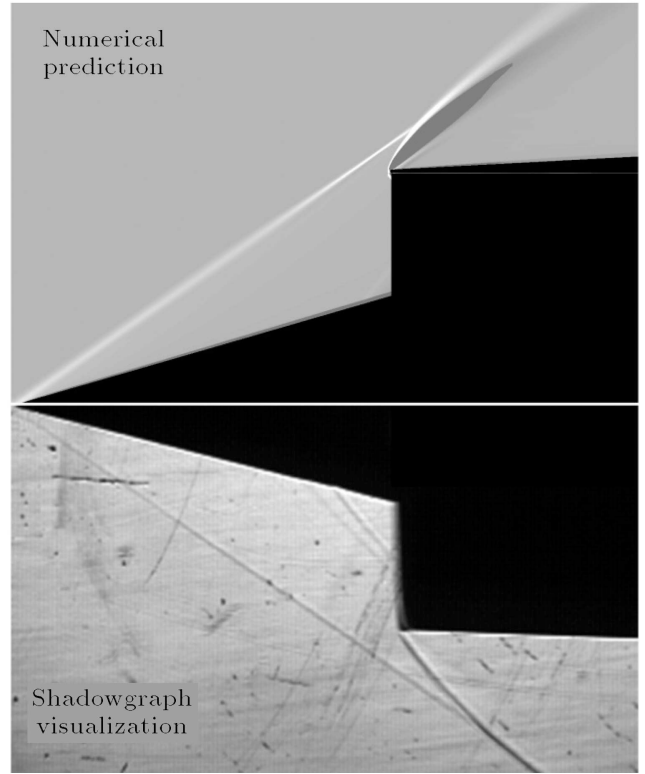

Figure 5. Comparison of numerical axial density gradient contour with experimental shadowgraph image, $M_{\infty}=2.0$ and $\alpha=0$.

the ratio of the mean total pressure at the end of the inlet duct to the free-stream total pressure. For the current inlet, mean total pressure at the end of the inlet duct is measured by the rake MR. Mass flow ratio, $\varepsilon$, is the ratio of captured mass flow rate to the mass flow rate in the case of full flow operation (no spillage) [2]. It is also defined as the ratio of free-stream to full flow captured streamtube area.

$$
\varepsilon=\frac{\dot{m}_{\infty}}{\dot{m}_{c}}=\frac{A_{\infty}}{A_{c}}
$$

Characteristic curve of the inlet under study at a freestream Mach number of 2.0, achieved by experimental pressure recordings, is shown in the left-hand side of Figure 6. As shown in this curve, three main operating 


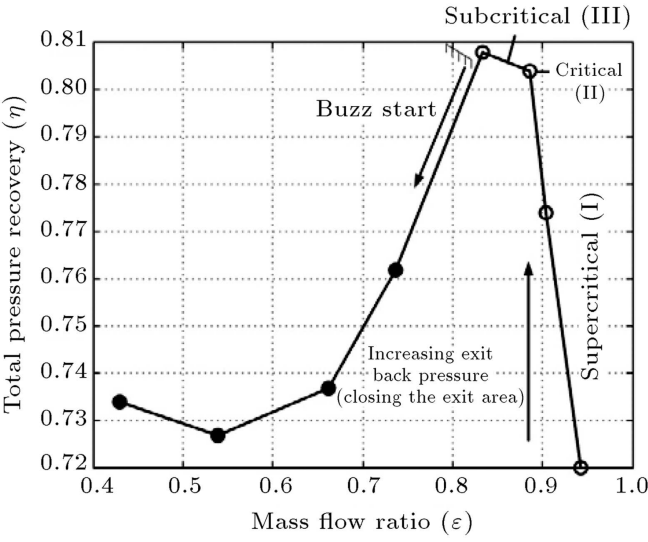

(a) Inlet characteristic curve

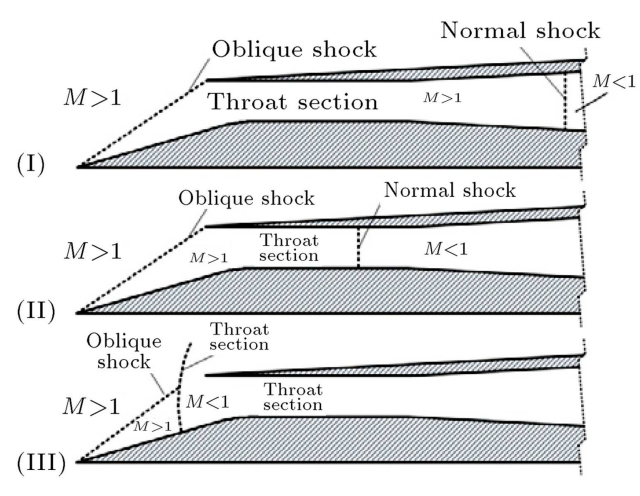

(b) Schematic of shock pattern

Figure 6. Experimental characteristic curve and operating regimes of mixed-compression inlet of this study and corresponding shock pattern at $M_{\infty}=2.0$.

regimes can be found for the inlet. These regimes were obtained through changing the inlet exit area by means of plug during the experiments. When the inlet exit area $\left(A_{e}\right)$ is open and the back pressure is low enough, the inlet operates at supercritical condition. Mass flow ratio is almost constant while back pressure ratio increases in this operating condition. The vertical region in the characteristic curve and the schematic (I) corresponds to this condition. According to schematic (I), normal shock is located in the divergent part of the diffuser for this operating region.

The terminal shock location is forced by applying the backpressure to the inlet (by combustion chamber in the real engine). In this case, increase in the surface pressure in the divergent part together with the adverse pressure gradient due to the shock makes the flow prone to separation and, consequently, low pressure recovery; also, high flow distortion occurs. As the exit area is further decreased, normal shock wave moves toward the throat until it stands in the throat and the critical operating regime happens. The best operating point of the inlet is the critical point as maximum MFR and TPR are achievable. Schematic (II) in Figure 6 shows the shock pattern relevant to this case. The nearly horizontal part in the curve, Figure 6(a), shows the critical operating condition. As seen in the curve, maximum amounts for both mass flow ratio and total pressure recovery are obtained in this case. By closing the inlet exit area further beyond the critical point, normal shock expels out of the inlet duct and flow spillage would happen; as a result, the inlet mass flow ratio deteriorates. In this case, interaction of the oblique and the normal shock is inevitable (schematic (III), Figure $6(\mathrm{~b})$. The flow would become unstable if the exit area is further decreased and the buzz phenomenon would be triggered. In this case, inlet performance decreases as it can be seen in characteristic curve of the inlet, Figure 6(a).

In all operating conditions of the inlet, interaction of shock with boundary-layer would greatly affect the flow field as well as the inlet performance. Physical aspects of SBLI in each operating region highly depend on the location of shock waves. According to this fact, types of flow separation in a mixed-compression inlet can be categorized. This task will be accomplished in the following section and the flow field corresponding to each category will be studied in the proceeding sections.

\section{Classification of SBLI for mixed-compression inlets}

For the mixed-compression inlets, compression is accomplished by a series of oblique shocks and a terminal normal shock. When operating in supercritical condition, a portion of supersonic compression occurs inside the inlet duct via an oblique shock train and a near-normal shock while in subcritical condition, all contraction takes place out of the inlet duct via one or several oblique shocks (depending on the ramp design) and terminates with a normal shock. There exist different types of flow interactions relevant to these different shock structures.

Figure 7 shows the grey-scale contour of axial derivative of density for the Exit Blockage Ratio (EBR) of $61 \%$ at a free-stream Mach number of 2.0 and at zero-degree angle of attack. It could be considered as the numerical Schlieren image of flow that is useful for understanding the shock structures inside the duct where experimental visualization of the flow is impossible. Comparing with schematics of Figure 6, it could be found that this figure is related to a supercritical operating condition. As Figure 7(a) demonstrates, the flow is compressed by an oblique shock before entering the duct. The external compression for the case study is done by a single conical shock due to the single-cone ramp. The external oblique shock in the case of full flow (critical and supercritical operating conditions at the design Mach number) impinges with the cowl lip 


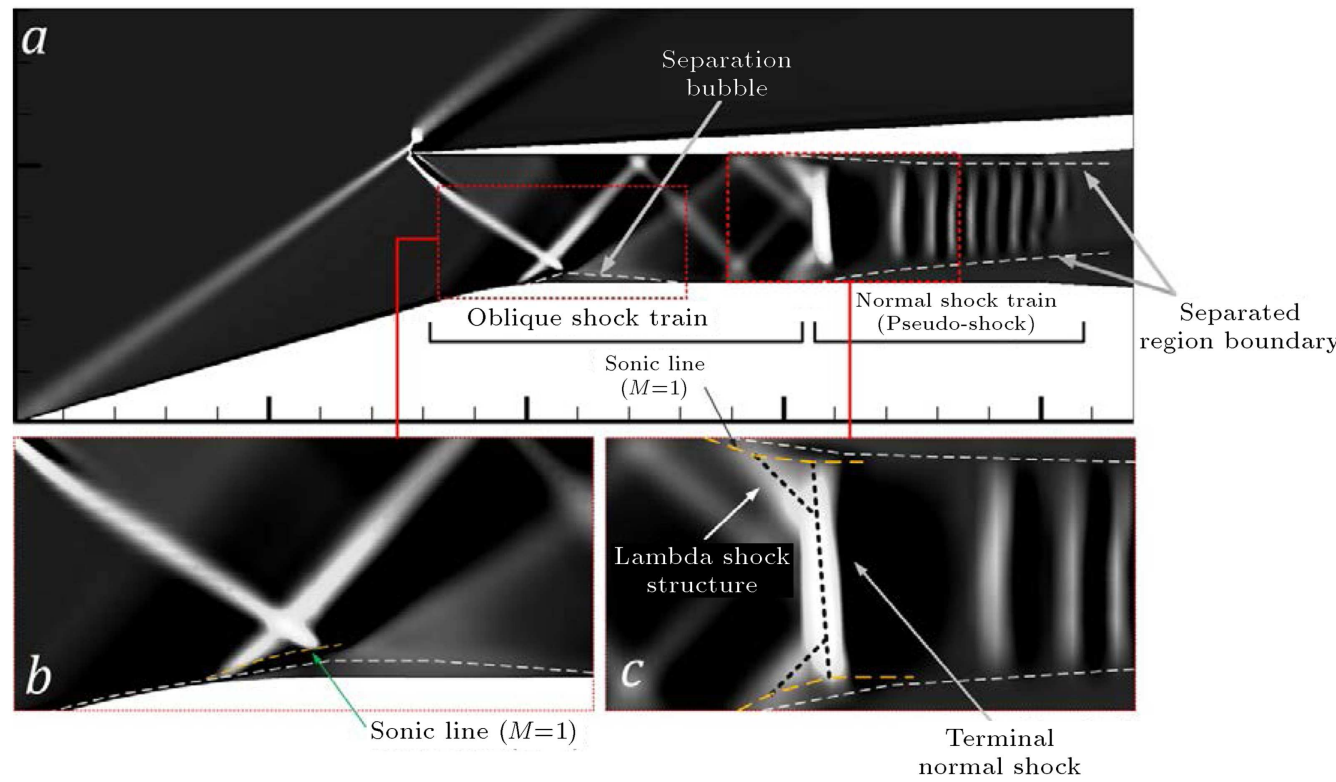

Figure 7. Numerical grey-scale contour of axial density gradient, $M_{\infty}=2.0, \alpha=0$, and $\mathrm{EBR}=61 \%$.

and is then reflected towards the spike surface. This incident shock separates the flow on the spike surface. Due to the formation of separated region, a separation shock forms and interacts with the reflected oblique shock.

Figure 7(b) depicts the enlarged picture of this oblique shock wave boundary-layer interaction zone. Separated flow reattaches downstream and a separation bubble is formed. An expansion fan and a reattachment shock wave are also produced to change the flow direction and to expand it. The flow then passes through consecutive reflections of oblique shock from both upper and lower walls of the duct and forms an oblique shock train. In the present paper, interactions of reflected oblique shocks with the boundary-layer together with the interactions of oblique shocks with each other are called oblique shock interactions. Details on the physical phenomena related to these interactions will be presented in the subsequent sections.

The flow passes terminal normal shock after passing the oblique shock train. If no boundary layer exists, the pressure would increase discontinuously by a single normal shock. However, in the real flow, adverse pressure gradient due to the shock separates the flow at normal shock foot and the shock structure gets the shape of lambda. Figure 7(c) illustrates the enlarged image of flow at the normal shock location. The flow becomes subsonic downstream of the normal shock, but the extent of separation increases. Flow is retarded in the separation region and the major part of the mass flow passes through the upper and lower separation zones (core region). As the effective flow area decreases, the flow accelerates again and becomes supersonic in the core region. In this case, flow acts like passing through a supersonic diffuser and a weaker normal shock is generated. This process continues; however, the generated shocks become weaker and the distance between them decreases. Finally, the flow becomes fully subsonic. The region of successive normal shocks, which is caused by the interaction of normal shock with boundary-layer, is called normal shock train in the literature [20].

The subsonic flow then goes through a mixing region where there is no shock; but pressure gradually increases to some extent. Static pressure in this region does not change along the vertical axis. The details for the flow in this region will be presented later in Section 7. The term pseudo-shock is used for the whole region of normal shock train and mixing region. Depending on the location of normal shock, or better say the inlet backpressure, the length of pseudo-shock may be different.

For the subcritical operating condition where the normal shock expels out of the inlet duct, interaction of normal shock with boundary-layer can be found as well. This interaction may or may not cause flow separation. Figure 8 shows the flow visualization

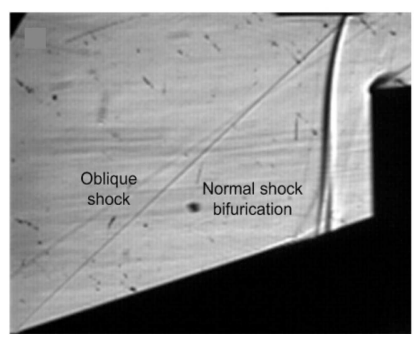

(a)

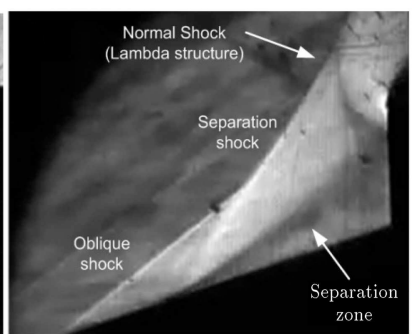

(b)
Figure 8. (a) Shadow graph flow visualization for: $M_{\infty}=2.0, \alpha=0$, and $\mathrm{EBR}=65 \%$. (b) Schlieren flow visualization for: $M_{\infty}=2.0, \alpha=0$, and $\mathrm{EBR}=70 \%$. 


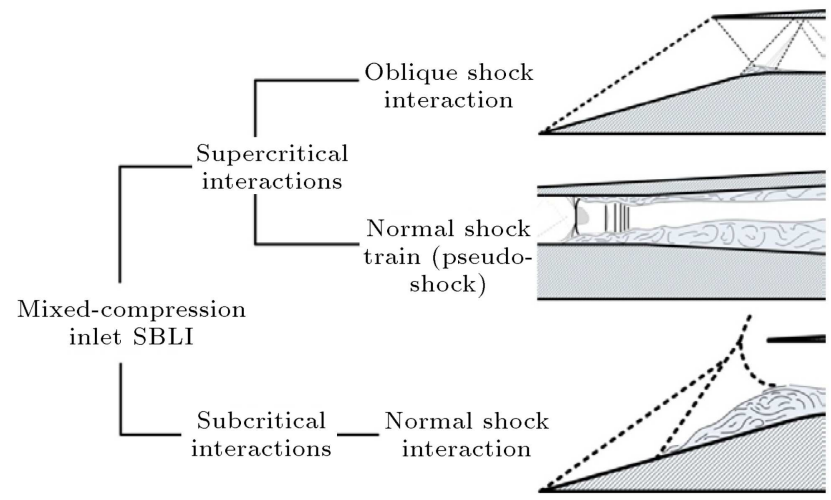

Figure 9. Classification of different SBLI flows related to a mixed-compression inlet.

images for 2 subcritical operation points. Figure 8(a) depicts the shock structure for the inlet at a freestream Mach number of 2.0 and an exit blockage ratio of $65 \%$. As shown in the figure, the normal shock is not strong enough to separate the flow, but there exist weak interactions and normal shock foot bifurcates. Yet, the flow in this case is stable. By further increasing the EBR, shock becomes stronger and the scale of separation grows. This causes the self-induced oscillations of shock. The phenomenon is called Buzz. Figure 8(b) illustrates the shock structure of flow in this case using a Schlieren image. Due to a greater flow separation, the angle of separationshock and its strength increase; consequently, shock bifurcation increases and normal shock gets the shape of lambda. Further study of flow physics in this case is considered in Section 8.

Therefore, interactions of shock with boundarylayers relevant to mixed-compression inlets can be classified into three major groups. Figure 9 indicates the classification chart for a mixed-compression inlet SBLI flow according to the operating conditions. Further discussion on each flow interaction phenomenon relevant to mixed-compression inlet on the basis of numerical and experimental results will be brought in the upcoming sections.

\section{Oblique shock interactions}

Figure 10 depicts variations of the wall static pressure ratio along the interaction zone together with the contour of Mach number for the present inlet at a freestream Mach number of 2.0 and for an Exit Blockage ratio of $60 \%$. The level of flood contour is bounded in order that the region of subsonic and supersonic flow can be divided and the sonic line becomes recognizable. As shown in this figure, a separation bubble forms downstream of the separation point S. A dividing streamline between points $\mathrm{S}$ and $\mathrm{R}$ can be seen that separates the recirculating bubble from the flow streaming from upstream to downstream. An energy

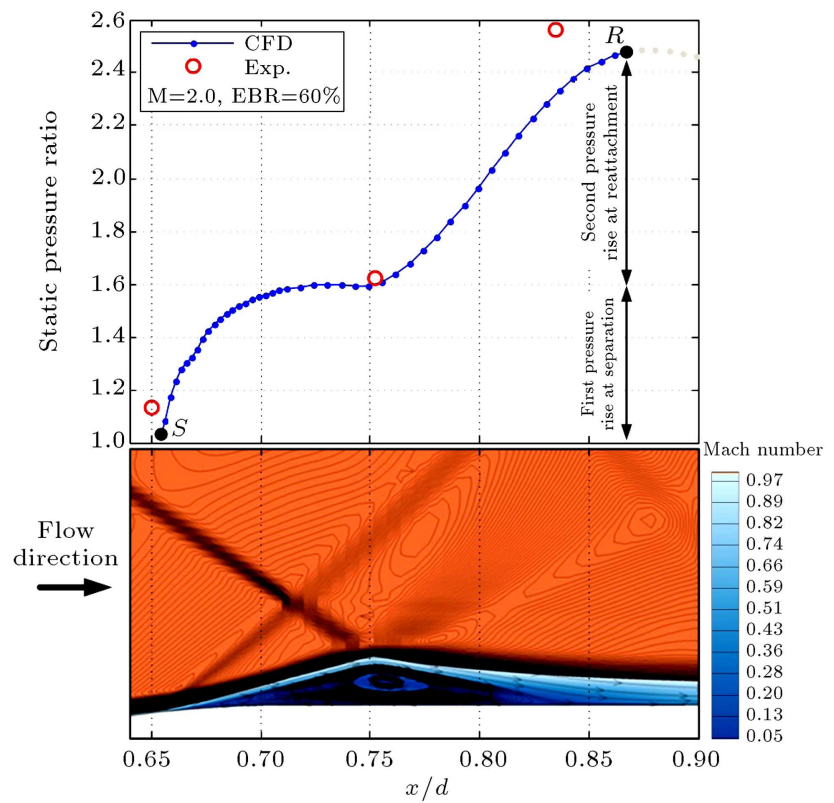

Figure 10. Wall pressure distribution in a shock-induced separated flow together with contour of Mach number for $M_{\infty}=2.0, \alpha=0$, and $\mathrm{EBR}=60 \%$.



Figure 11. Sketch of flow induced by a shock reflection with separation.

transfer takes place from high-speed flow outside the bubble towards the separated inner region. This fact causes the flow velocity to increase as they approach downstream and reattaches the streamline [1]. Due to the strong interaction of shock with boundary-layer, this inner region is highly affected by the viscosity. As it is illustrated in Figure 10, specific shock patterns are shaped by this interaction.

Figure 11 shows a schematic of the flow induced by a shock reflection with separation. A separation shock is located upstream of point $\mathrm{S}$ and deflects the flow direction upward. This separation shock interacts with the incident shock. The slope of the separation bubble is smooth; hence, the compression near the surface is performed by several compression waves. In the reattachment part, the direction of flow should be changed downward; therefore, an expansion fan is formed. Downstream of the separation bubble, the flow 
direction becomes parallel to the surface by means of a reattachment shock. As seen in Figure 11, incident shock penetrates the rotational inviscid part of the boundary-layer and bends due to the reduction of local Mach number. The intensity of shock weakens and vanishes when it reaches the boundary-layer sonic line.

The wall pressure distribution of Figure 10 initially exhibits a steep rise, associated with separation. Another more progressive pressure rise is seen to occur during the reattachment. It is found from the pressure distribution that there exists considerable deviation from sudden pressure jump of the pure inviscid solution, Figure 10. The reason is that in the strong shock interactions, the viscosity plays a key role in the flow structure. Subsequent oblique shocks, reflected from upper and lower walls of the inlet duct, also interact with boundary-layer when impinging with the opposite surface; but the interactions become weaker and no separation would occur.

However, the question is that how such an interaction affects the inlet performance? Figure 12 shows numerical results for the radial distribution of total pressure ratio across the separation zone for $\mathrm{EBR}=$ $61 \%$. Shaded region indicates the total pressure losses in comparison with the inviscid shock reflection. It is found from this figure that interaction of oblique shock with spike boundary-layer creates a region of dead flow and reduces the total pressure of the inlet. At the end of the first pressure rise zone, total pressure loss is maximum due to the extent of the flow separation in that position. Also, interactions of shock waves with the boundary-layer of the internal surface of inlet cowl result in some total pressure losses, Figure 12. Using some means of boundary-layer removal would help avoiding the total pressure losses.

\section{Normal shock train and pseudo-shock}

Figure 13 shows contour of the axial density derivative together with the static pressure distribution on the

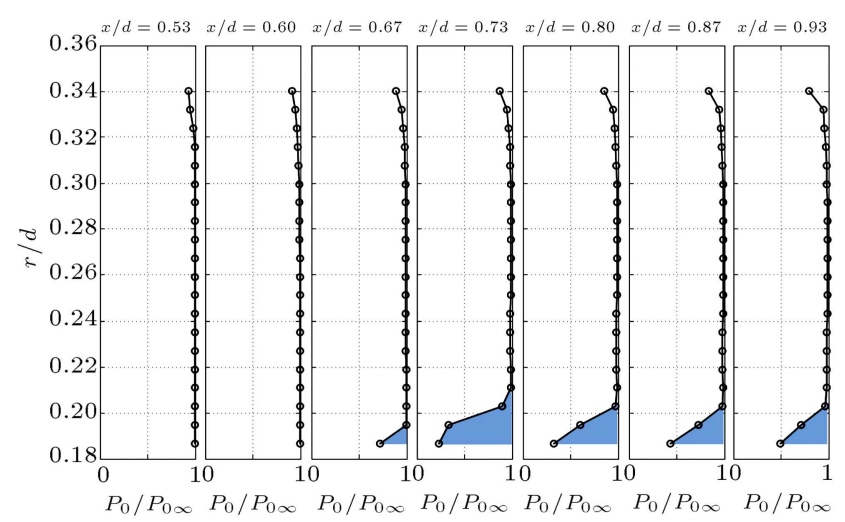

Figure 12. Numerical results for radial distribution of total pressure ratio across the interaction zone, $\mathrm{EBR}=61 \%$.

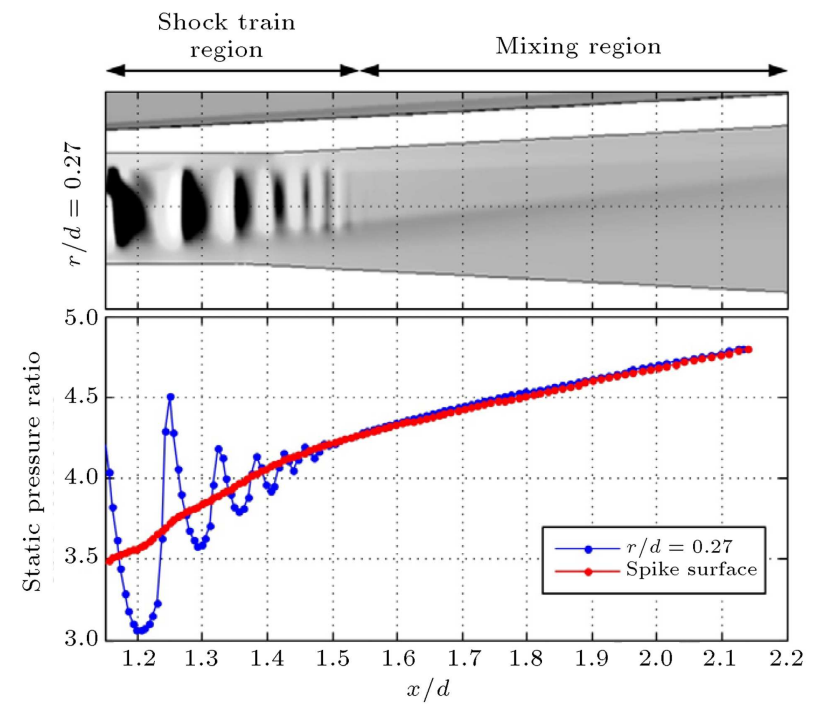

Figure 13. Numerical wall pressure distribution and pressure distribution across a horizontal line together with the corresponding contour of axial density derivative, $M_{\infty}=2.0, \alpha=0$, and $\mathrm{EBR}=60 \%$.

wall and in the middle line of the duct for the present mixed-compression inlet at a free-stream Mach number of 2.0 and for Exit blockage ratio of $60 \%$. A normal shock train inside the duct appears. Dark regions behind the shocks demonstrate subsonic region. It could be found that the flow behind the shock goes through subsonic to supersonic isentropically and again decelerates to subsonic speed through a subsequent normal shock. Pressure increases sharply through each shock and then decreases behind the shock until it reaches the downstream normal shock. It is found from either the pressure distribution or the contour that the strength of shocks decreases as they approach downstream. The region in which the shocks are located is called the shock train region. After the last shock, the flow becomes subsonic, but there exists velocity difference between the core flow and the nearwall region. As a result, a momentum exchange process between the core and the inner region flow takes place that makes the flow fully developed at the end of the region. The static pressure becomes constant along the radial direction, as seen in Figure 13, but the velocity profile changes continuously until the flow becomes uniform.

Figure 14 depicts axial density derivative for the mixed-compression inlet at different back pressure ratios for a free-stream Mach number of 2.0. For low back pressure ratios, most of the compression is done by oblique shock train and a single normal shock is seen. In this case, separated flow at the engine face highly affects the flow distortion. Increasing the backpressure ratio pushes the terminal normal shock upstream and the normal shock train forms. For moderate EBRs, the length of normal shock train and 


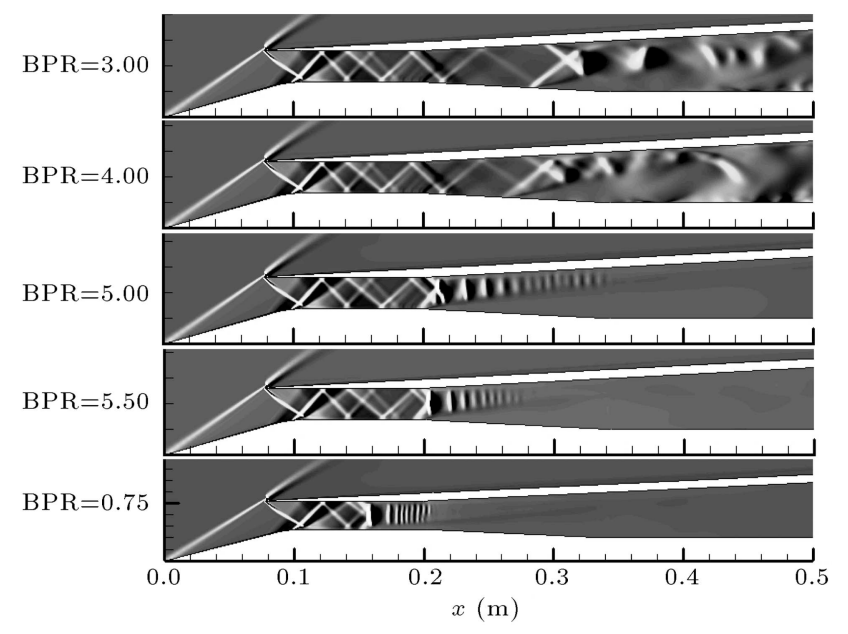

Figure 14. Effect of back pressure ratio on the numerical contour of axial density derivative, $M_{\infty}=2.0$.

the mixing region as well as the number of consecutive shocks is large. As EBR further increases, the length of pseudo-shock decreases. If the length of pseudo-shock becomes smaller in comparison with the throat length, the amount of total pressure loss decreases.

Figures 15 demonstrates experimental measurements for total pressure recovery and flow distortion, respectively, at rake $\mathrm{MR}$ for different exit blockage ratios at free-stream Mach number of 2.0 and at zerodegree angle of attack. For cases with buzz, the time average of pressure signal is used. For EBR = $50 \%$, variation of the total pressure across the radial direction is high since the terminal shock is located aft and the separated flow region is thicker. As the blockage ratio increases, the terminal shock moves upstream and the separated region becomes thinner and total pressure increases. For $\mathrm{EBR}=62.5 \%$, it is seen that a near-constant total pressure distribution is achieved, Figure 15(a). The flow distortion for this blockage ratio is also lower; however, a small deviation from the uniform distribution near the upper wall occurs. Having performance curve of Figure 6 and shadowgraph image of Figure 8 (a) in mind, we know that at $\mathrm{EBR}=65.0 \%$, the present inlet performs in the subcritical condition. Therefore, the separation due to the terminal normal shock is completely eliminated at the engine face and as a result, a more uniform stagnation pressure distribution is achieved as shown in Figure 15(a) for EBR $=65 \%$. For the subsequent subcritical operating conditions, the flow distortion remains rather low. Losses due to buzz phenomenon and flow separation on the compression surface cause the amount of stagnation pressure to reduce as the inlet mass flow ratio decreases.

Figure 16 shows the pressure spectra at $x / d=$ 2.4 measured by rake MR for different exit blockage ratios before buzz onset at a free-stream Mach number of 2.0 and at zero-degree angle of attack. For EBRs equal to $55.0 \%$ and $60.0 \%$ at measured pressure spectra, this figure indicates high magnitude at almost all frequencies, which is a typical behavior of separated flow. An important point is that the amplitude of pressure signal at different frequencies for $\mathrm{EBR}=60.0 \%$ is lower than the one for $\mathrm{EBR}=$ $55.0 \%$. As the terminal normal shock moves upstream through increasing the back pressure, the pseudo-shock approaches upstream and its length decreases. As a result, the momentum exchange between central core flow and low-speed dissipative flow starts earlier for greater EBRs and because of this oscillations of flow near the wall, it decreases. For higher exit blockage ratios, one cannot find any considerable magnitudes at high and at moderate frequencies. This is another reason for the trend of flow distortion that is illustrated in Figure 15(b).

Therefore, it is seen that the pseudo-shock highly affects inlet performance parameters, especially flow distortion, at the engine face. On the other hand, pseudo-shock has some dynamic distortion effects that stem from the self-excited oscillations of shock-train.

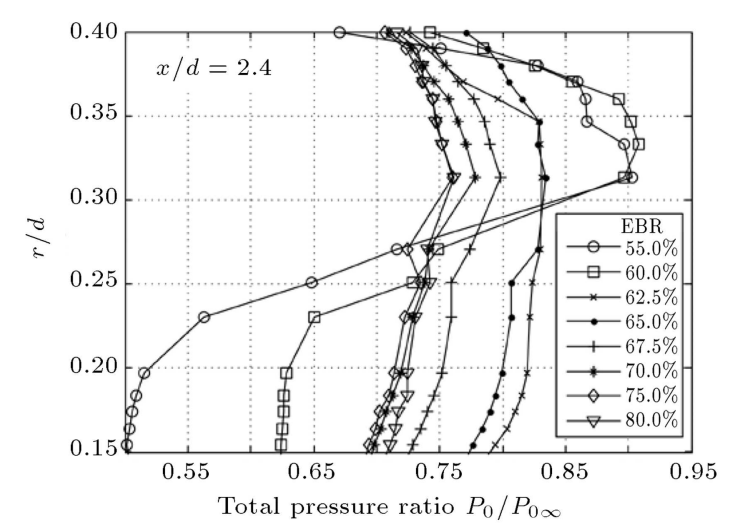

(a)

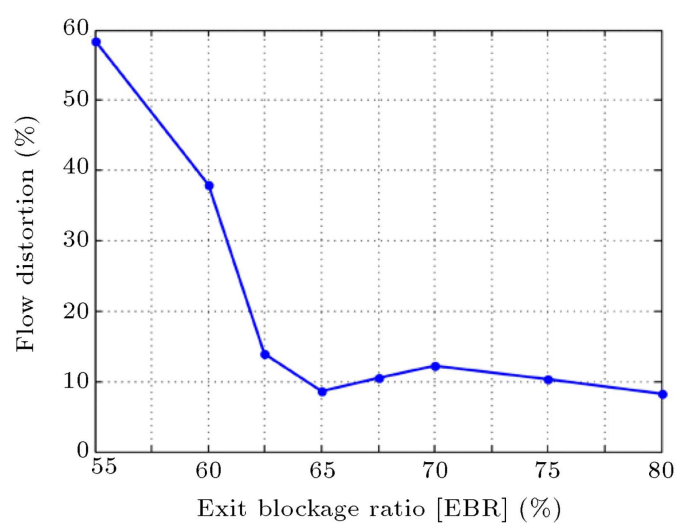

(b)

Figure 15. (a) Experimental radial total pressure distributions at rake MR at different EBRs, $M_{\infty}=2.0$ and $\alpha=0$. (b) Experimental flow distortions at rake MR for different EBRs at $M_{\infty}=2.0$ and $\alpha=0$. 


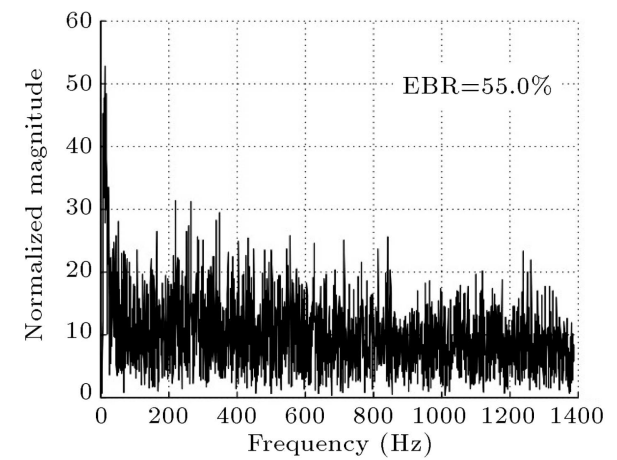

(a)

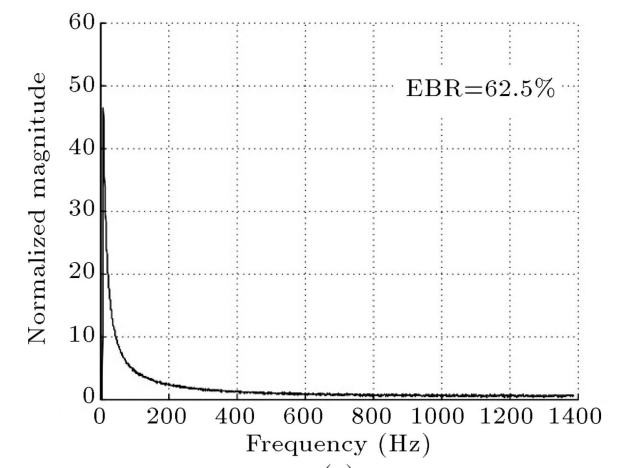

(c)

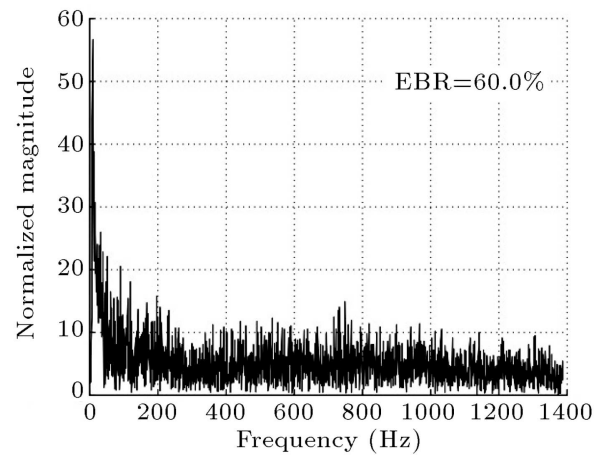

(b)



(d)

Figure 16. Spectra of measured wall pressure for different exit blockage ratios at $x / d=2.4, M_{\infty}=2.0$, and $\alpha=0$.
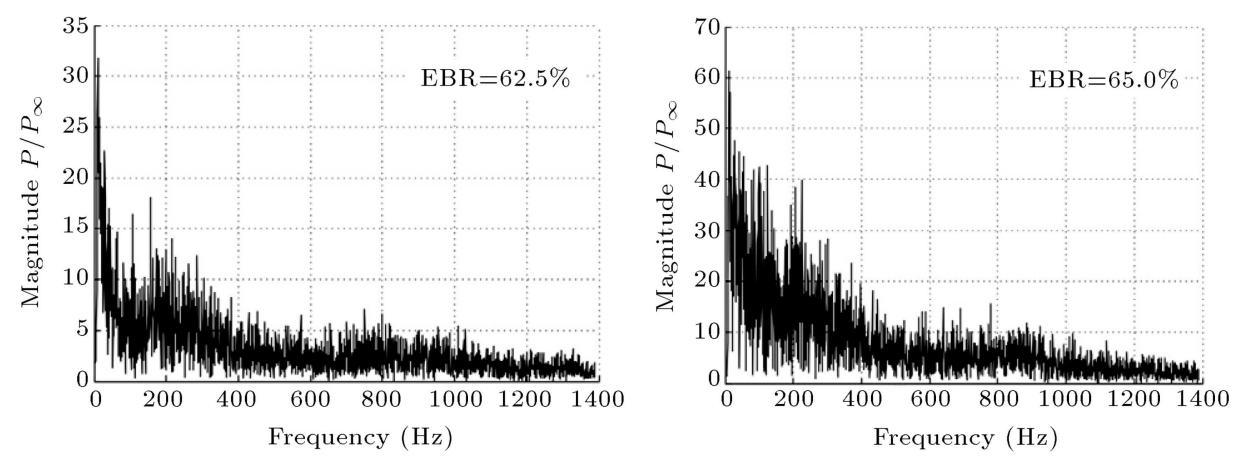

Figure 17. Spectra of measured total pressure for two EBRs at $r / d=0.27, x / d=2.4, M_{\infty}=2.0$, and $\alpha=0$.

When shock interacts with boundary-layer, the location of shock cannot be determined easily since it fluctuates with time, even if boundary conditions are held constant upstream and downstream of the shock.

Figure 17 shows spectra of total pressure measured at $x / d=2.4$ and in the centreline of inlet duct $(r / d=0.27)$ for a free-stream Mach number of 2.0. Wall pressure spectra for EBR $=62.5 \%$ and $\mathrm{EBR}=65.0 \%$, as shown in Figure 16, has no considerable oscillation frequencies. Furthermore, total pressure distribution of Figure 15(a) confirms that the width of retarded flow region is far less than the lower exit blockage ratios. Therefore, the flow in the core region at these exit blockage ratios is expected to be uniform while the upstream and downstream boundary conditions are constant. However, as Figure 17 illustrates, several active frequencies can be found in the total pressure spectra of stagnation pressures. The reason may be oscillations of the pseudo-shock that cause the downstream total pressure to fluctuate. The strength of normal shock trains may differ as they move upstream and downstream. In correlation with the shock strength, total pressure loss due to shock differs and makes the total pressure at the end of the inlet duct change. Meanwhile, no distinct frequency can be detected to assign for the pseudo-shock oscillations.

Total pressure tap of Figure 17 is located nearly at the end of inlet duct. At EBR $=62.5 \%$ and $65.0 \%$, pressure tap is located at the end of mixing region of pseudo-shock and in the separated flow of spike surface. Thus, oscillations of pseudo-shock structure do not lead to an alternating total pressure change in this region, but inject some oscillatory perturbations into the mixing region. This leads to a set of random 
oscillations and in this way, a set of frequencies with considerable magnitude can be found in the pressure spectra. The spectra of $\mathrm{EBR}=65.0 \%$ involve more frequencies with considerable magnitude in comparison with the $\mathrm{EBR}=62.5 \%$, while the flow is expected to be less oscillatory due to elimination of the pseudoshock at $\mathrm{EBR}=65.0 \%$. The expelled normal shock for $\mathrm{EBR}=65.0 \%$ interacts with the boundary-layer of external compression cone that leads to little buzz oscillations. This flow phenomenon is discussed in the next section.

\section{Subcritical interactions}

As the inlet back pressure increases, the upstream normal shock is pushed until it expels out of the inlet duct. In this case, interaction of normal shock with boundary-layer occurs. For moderate back pressure ratios, the interaction is weak (Figure 8(a)). Further reduction of exit area strengthens the normal shock and the boundary-layer becomes prone to separation.

Figure 18 shows schematic of the normal SBLI together with a Schlieren image of flow field for the present mixed-compression inlet. Separation zone acts like a viscous wedge and a separation shock forms. This shock interacts with normal shock in a point known as triple point and a slip line emits from the point of interaction across which the flow velocity changes sharply. Shock foot declines near the separation zone and creates a lambda-like structure. The separation zone grows inside the duct and results in flow spillage. In this way, separation blocks the flow direction and increases the inlet back pressure. It is found to be one of the mechanisms of Buzz initiation known as Dailey criterion [40]. This mechanism results in high amplitude oscillations of normal shock and affects the inlet flow stability.

Another buzz mechanism was found by Ferri and Nucci in 1951 [41]. They concluded that buzz starts when vortex sheet, which stems from interaction of

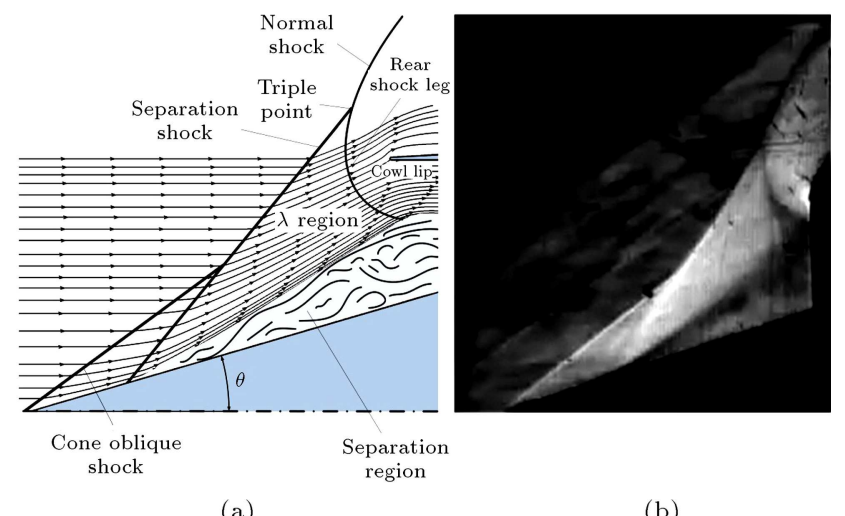

(a)

(b)

Figure 18. (a) Schlieren image of flow field. (b) Schematic of normal SBLI of mixed-compression inlet.

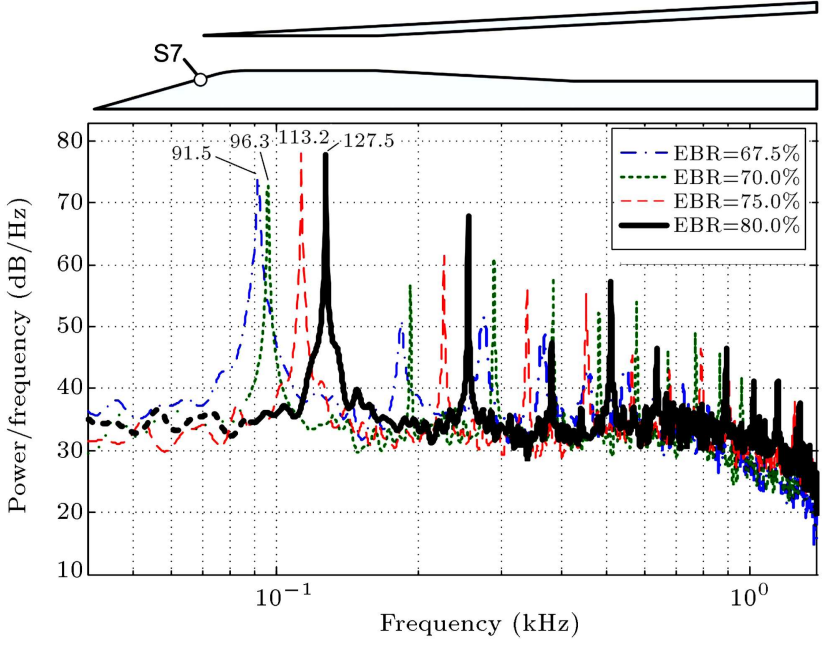

Figure 19. Power spectral density of sensor S7 at different EBR values, $x / d=0.374, M_{\infty}=2.0$, and $\alpha=0$.

shock waves, collides with the cowl inner surface. Velocity discontinuity across the vortex sheet causes the flow on internal surfaces of the duct to separate and diffuser chocking results. This mechanism leads to low-amplitude high-frequency oscillations of normal shock wave.

For the present inlet, the dominant mechanism is Dailey criterion. Figure 19 shows the power spectral density of pressure recording for a tap on the external compression cone surface. It is seen that significant frequencies are detectable in relation with buzz for various EBRs. The fundamental frequency of buzz increases as the exit blockage ratio increases. Note that as the mass flow ratio reduces the strength of the shock, interaction increases. To better realize the behavior of boundary-layer during a buzz cycle, Schlieren images of the flow field are provided in Figure 20. This figure shows four consecutive instants in a big buzz cycle (high-amplitude oscillation). The time interval between each image is $1 \mathrm{~ms}$. It is seen that as the time interval increases, the separation zone grows and pushes the upstream normal shock towards the incoming flow. Extent of the separation region is seen

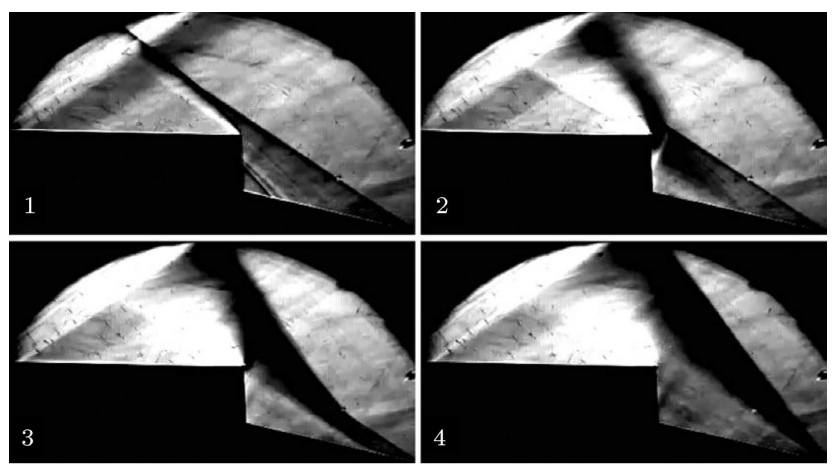

Figure 20. Schlieren images of four consecutive instants of buzz: $M_{\infty}=2.0, \alpha=0$ and $\mathrm{EBR}=75.0 \%$. 
to increase and when the normal shock is positioned in the most upstream location, $t=4 \mathrm{~ms}$, the inlet is completely blocked. This figure shows the great strength of SBLI for the present inlet. Therefore, it can be concluded that application of flow control by any means, such as the boundary-layer suction, is essential for this inlet.

\section{Conclusion}

This study reveals the existence of various shock wave boundary-layer interactions relevant to the supersonic inlets. Results show that these flow interaction phenomena have different effects on the stability and on the performance of the inlet. Interaction of terminal normal shock with internal duct boundary-layer causes pseudo-shock phenomenon that leads to increase in flow distortion and reduction of total pressure recovery. In addition, interaction of normal shock wave with external cone boundary-layer causes buzz instability and degrades inlet performance. The unsteady nature of flow due to shock boundary-layer interactions is also detected by means of experimental measurements. In supercritical operating condition, pseudo-shock has an oscillatory nature that may cause dynamic distortion at the engine face. In subcritical operating condition that normal shock resides on the external compression cone, normal shock interacts with boundary-layer in that region. This interaction, if the back pressure ratio is high enough, leads to flow separation and may even chock the inlet. It causes self-excited flow oscillations, called buzz, and specific frequencies can be found for oscillations in the measured pressure spectra. Flow stability of inlet highly depends on the buzz characteristics. In order to avoid harmful effects of such interactions, flow control devices such as boundarylayer bleed should be applied.

Consequently, behavior of SBLI flow phenomena should be understood carefully in order to consider the corresponding effects of interactions during the inlet design process and for designing flow control devices.

\section{Acknowledgement}

The authors gratefully acknowledge the supports of the Mesbah Research Institute.

\section{Nomenclature}

\section{Latin letter}

$A_{c} \quad$ Inlet capture area $\left(\mathrm{m}^{2}\right)$

$A_{\infty} \quad$ Captured free stream area $\left(\mathrm{m}^{2}\right)$

$d \quad$ Intake maximum outer diameter $(\mathrm{m})$

L Intake length $(\mathrm{m})$

\begin{tabular}{|c|c|}
\hline$\dot{m}$ & Mass flow rate through $\mathrm{i}$ \\
\hline $\mathrm{M}$ & Mach number \\
\hline$P$ & Pressure $(\mathrm{Pa})$ \\
\hline \multicolumn{2}{|c|}{ Greek letter } \\
\hline f & Mass flow ratio, $A_{\infty} / A_{c}$ \\
\hline$\eta$ & Pressure recovery \\
\hline$\alpha$ & Angle of attack (deg) \\
\hline$\phi$ & Rotational angle (deg) \\
\hline$\theta$ & Cone angle (deg) \\
\hline \multicolumn{2}{|c|}{ Subscripts } \\
\hline 0 & Total conditions \\
\hline & Free stream conditions \\
\hline
\end{tabular}

\section{References}

1. Babinsky, H. and Harvey, J.K., Shock Wave-BoundaryLayer Interactions, Cambridge University Press, New York, USA (2011).

2. Seddon, J. and Goldsmith, E.L., Intake Aerodynamics, AIAA Education Series, 2nd Edn., pp. 268-292, AIAA, Reston, VA, USA (1999).

3. Trapier, S., Duveau, P. and Deck, S. "Experimental study of supersonic inlet buzz", A IA A Journal, 44(10), pp. 2354-2356 (2006).

4. Trapier, S., Deck, S. and Duveau, P. "Time-frequency analysis and detection of supersonic inlet buzz", AIA A Journal, 45(9), pp. 2273-2284 (2007).

5. Herrmann, D. and Triesch, K. "Experimental investigation of isolated inlets for high agile missiles", Aerospace Science and Technology, 10(8), pp. 659-667 (2006).

6. Hirschen, C., Herrmann, D. and Gulhan, A. "Experimental investigations of the performance and unsteady behavior of a supersonic intake", Journal of Propulsion and Power, 23(3), pp. 566-574 (2007).

7. Herrmann, D., Triesch, K. and Gulhan, A. "experimental study of chin intakes for airbreathing missiles with high agility", Journal of Propulsion and Power, 24(2), pp. 236-244 (2008).

8. Das, S. and Prasad, J.K. "Unstart suppression and performance analysis of supersonic air-intake adopting bleed and cowl bending", Journal of the Institution of Engineers (India)-Aerospace Engineering, 9(1), pp. $27-35$ (2010).

9. Soltani, M.R., Farahani, M. and Asgari Kaji, M.H. "An experimental study of buzz instability in an axisymmetric supersonic inlet", Scientia Iranica, 18(2), pp. 241-249 (2011).

10. Herrmann, D., Blem, S. and Gulhan, A. "Experimental study of boundary-layer bleed impact on ramjet inlet performance", Journal of Propulsion and Power, 27(6), pp. 1186-1195 (2011). 
11. Soltani, M.R. and Farahani, M. "Experimental investigation of effects of Mach number on the flow instability in a supersonic inlet", Experimental Techniques, 37(3), pp. $46-54$ (2013).

12. Soltani, M.R. and Farahani, M. "Effects of angle of attack on the inlet buzz", Journal of Propulsion and Power, 28(4), pp. 747-757 (2012).

13. Herrmann, D., Siebe, F. and Gulhan, A. "Pressure fluctuations (buzzing) and inlet performance of an airbreathing missile", Journal of Propulsion and Power, 29(4), pp. 839-848 (2013).

14. Soltani, M.R., Sepahi Younsi, J. and Daliri, A. "Performance investigation of a supersonic air intake in the presence of the boundary-layer suction", Proc. IMechE Part G: J. Aerospace Engineering, 229(8), pp. 14951509 (2015).

15. Lu, P.J. and Jain, L.T. "Numerical investigation of inlet buzz flow", Journal of Propulsion and Power, 14(1), pp. 90-100 (1998).

16. Oh, J.Y., Ma, F., Hsieh, S.Y. and Yan V. "Interactions between shock and acoustic waves in a supersonic inlet diffuser", Journal of Propulsion and Power, 21(3), pp. 486-495 (2005).

17. Trapier, S., Deck, S. and Duveau, P. "Delayed detached-eddy simulation and analysis of supersonic inlet buzz", AIA A Journal, 46(1), pp. 118-131 (2008).

18. Vivek, P. and Mittal, S. "Buzz instability in a mixedcompression air intake", Journal of Propulsion and Power, 25(3), pp. 819-822 (2009).

19. Kotteda, V.M.K. and Mittal, S. "Viscous flow in a mixed compression intake", Int. J. Numer. Meth. Fluids, 67(11), pp. 1393-1417 (2011).

20. Viswanath, P.R. "Shock-wave turbulent boundarylayer interaction and its control: a survey of recent development", Sadhana, 12(1), pp. 45-104 (1988).

21. Matsuo, K., Miyazato, Y. and Kim, H.D. "Shock train and pseudo-shock phenomena in internal gas flows", Progress in Aerospace Sciences, 35(1), pp. 33100 (1999).

22. Knight, D., Yan, H., Panaras, A.G. and Zheltovodov, A. "Advances in CFD prediction of shock wave turbulent boundary layer interactions", Progress in Aerospace Sciences, 39(2-3), pp. 121-184 (2003).

23. Mousavi, S.M. and Roohi, E. "Large eddy simulation of shock train in a convergent-divergent nozzle", International Journal of Modern Physics C, 25(4), p. 1450003 (2014).

24. Mousavi, S.M. and Roohi, E. "Three dimensional investigation of the shock train structure in a convergentdivergent nozzle", Acta Aeronautica, 105(1), pp. 117127 (2014).

25. Gaitonde, D.V. "Progress in shock wave/boundary layer interactions", AIAA 43rd Fluid Dynamics Conference, San Diego, CA, AIAA Paper 2013-2607 (2013).
26. Dussauge, J.P., Dupont, P. and Debieve, J.F. "Unsteadiness in shock wave boundary layer interactions with separation", Aerospace Science and Technology, 10(2), pp. 85-91 (2006).

27. Aubard, G., Gloerfelt, X. and Robinet, J.C. "Largeeddy simulation of broadband unsteadiness in a shock/boundary-layer interaction", AIAA Journal, 51(10), pp. 2395-2409 (2013).

28. Galli, A., Corbel, B. and Bur, R. "Control of forced shock-wave oscillations and separated boundary layer interaction", Aerospace Science and Technology, 9(8), pp. 653-660 (2005).

29. Titchener, N. and Babinsky, H. "Shock wave/boundary-layer interaction control using a combination of vortex generators and bleed", AIA A Journal, 51(5), pp. 1221-1233 (2013).

30. Ali, Y.M., Alvi, F.S. and Kumar, R. "Studies on the influence of steady microactuators on shockwave/boundary-layer interaction", AIAA Journal, 51(12), pp. 2753-2762 (2013).

31. Hamed, A. and Shang, J.S. "Survey of validation data base for shockwave boundary-layer interactions in supersonic inlets", Journal of Propulsion and Power, 7(4), pp. 617-625 (1991).

32. Sajben, M., Donovan, J.F. and Morris, M.J. "Experimental investigation of terminal shock sensors for mixed-compression inlets", Journal of Propulsion and Power, 8(1), pp. 168-174 (1992).

33. Fischer, C. and Olivier, H. "Experimental investigation of the shock train in an isolator of a scramjet inlet", 17th AIA A International Space Planes and Hypersonic Systems and Technologies Conference, San Francisco, CA, AIAA 2011-2220 (2011).

34. Troia, T.J., Patel, A.A., Crouse, D. and Hall, G.R. "Passive device flow control for normal shock/boundary layer interactions in external compression inlets", 41st AIAA Fluid Dynamics Conference and Exhibit, Hawaii, Honolulu, AIAA 2011-3911 (2011).

35. Loth, E., Titchener, N., Babinsky, H. and Povinelli, L. "Canonical normal shock wave/boundary-layer interaction flow relevant to external compression inlets", AIAA Journal, 51(9), pp. 2208-2217 (2013).

36. Settles, G.S., Schlieren and Shadowgraph Techniques, Springer-Verlag, New York, USA (2001).

37. Soltani, M.R., Sepahi Younsi, J. and Farahani, M. "Investigation of new flux scheme for the numerical simulation of the supersonic intake flow", Proc. IMechE Part G: J. Aerospace Engineering, 226(11), pp. 1445-1454 (2011).

38. Kwak, E. and Lee, S. "Convergence study of inlet buzz frequency with computational parameters", 29th AIAA Applied Aerodynamics Conference, Hawaii, Honolulu, AIAA 2011-3362 (2011).

39. Menter, F.R. "Two-equation eddy-viscosity turbulence 
models for engineering applications", AIA A Journal, 32(8), pp. 1598-1605 (1994).

40. Dailey, C.L. "Supersonic diffuser instability", PHD Thesis, California Institute of Technology, USA (1954).

41. Ferri, A. and Nucci, L.M. "The origin of aerodynamics instability of supersonic inlet at subcritical condition", NACA RM L50K30 (1951).

\section{Biographies}

Mohammad Reza Soltani received his $\mathrm{PhD}$ degree in Aerodynamics from the University of Illinois at Urbana-Champaign, USA, and is now Professor in the Aerospace Engineering Department at Sharif University of Technology, Tehran. His research interests include applied aerodynamics, unsteady aerodynamics, wind tunnel testing, wind tunnel design, and data processing.
Abbas Daliri received his BS degree in Mechanical Engineering from Ferdowsi University of Mashhad, Mashhad, Iran. He has MS in Aerodynamics from the Aerospace Engineering Department at Sharif University of Technology, Tehran, Iran. He is now a PhD candidate of Aerodynamics at Ferdowsi University of Mashhad, Mashhad, Iran. His field of interest is experimental aerodynamics and fluid mechanics and wind tunnel testing.

Javad Sepahi Younsi was born in 1985 in Younsi, Gonabad, Iran. He received his $\mathrm{MS}$ and $\mathrm{PhD}$ degrees in Aerodynamics from the Aerospace Engineering Department at Sharif University of Technology, Tehran, Iran. He is now Assistant Professor in the Mechanical and Aerospace Engineering Department at Ferdowsi University of Mashhad, Mashhad, Iran. He works in the field of numerical and experimental aerodynamics. 